\title{
International Journal of Sciences
}

\section{Monitoring of Elemental Contamination in Groundwater Samples of Sobhodero Khairpur, Sindh, Pakistan}

\section{Mushtaque Ali Jakhrani', Muhammad Qasim², Shahid Ali Jakhrani1 ${ }^{1}$, Khan Muhammmad Malik¹, Farkhanda Zaman Dayo², Sahrish Fareed Memon ${ }^{1}$}

\author{
${ }^{1}$ Institute of Chemistry, Shah Abdul Latif University, Khairpur, Sindh, Pakistan \\ ${ }^{2}$ Institute of Chemical Sciences, Bahauddin Zakariya University, Multan, Pakistan \\ ${ }^{2}$ Department of Zoology, Shah Abdul Latif University, Khairpur, Sindh, Pakistan
}

\begin{abstract}
The aim of present study was to monitor arsenic and other trace and toxic elemental exposure in groundwater ofT aluka Sobhodero being most populous Taluka of District Khairpur, Sindh, Pakistan. 333 groundwater samples were collected on the basis of Union Councils throughout Taluka Sobhodero. Among 333 samples, 90 were collected from tube well (90-TW) and 243 were collected from hand pump (243-HP) sources in the study area. Atomic Absorption Spectroscopy (Perkin Elmer, AAS-100) was used for analysis of elemental concentrations but in case of arsenic analysis AAS coupled with mercury hydride generator MHS-15 was used in the laboratories of Institute of Chemistry, Shah Abdul Latif University, Khairpur, Pakistan. The concentrations of arsenic, copper, iron, nickel, lead and zinc were found in range of $19.5-58 \mu \mathrm{gL}^{-1}, 85-260 \mu \mathrm{gL}^{-1}, 209-412 \mu \mathrm{gL}^{-1}, 01-$ $19 \mu \mathrm{gL}^{-1}, 06-14 \mu \mathrm{gL}^{-1}$ and $114-420 \mu \mathrm{gL}^{-1}$ respectively in $\mathrm{HP}^{-1}$ samples and8.6-36 $\mu \mathrm{gL}^{-1}, 16-90 \mu \mathrm{gL}^{-1}, 45-100 \mu \mathrm{gL}^{-1}, 01-$ $09 \mu \mathrm{gL}^{-1}, 03-08 \mu \mathrm{gL}^{-1}$ and $22-111 \mu \mathrm{gL}^{-1}$ correspondingly in TW samples. The proposed maximum contamination limit (MCL) for $\mathrm{As}, \mathrm{Cu}, \mathrm{Fe}, \mathrm{Ni}, \mathrm{Pb}$ and $\mathrm{Zn}$ in drinking water was10, 2000,300,20, 100, and $3000 \mu \mathrm{gL}^{-1}$ respectively as specified by WHO. The comparative study indicated that groundwater samples collected from TW sources have shown lowest levels of $\mathrm{As}, \mathrm{Cu}, \mathrm{Fe}, \mathrm{Ni}, \mathrm{Pb}$ and $\mathrm{Zn}$ as compared to $\mathrm{HP}$ samples possibly due to higher depths of the motor pumps.
\end{abstract}

Keywords: Arsenic; Toxic metals, Drinking water, Atomic Absorption Spectrometry

\section{Introduction}

Water is an essential component for survival of life on earth. It contains important minerals for humans as well as for the organisms living on earth and aquatics. Contamination of drinking water especially with toxic elements and arsenic is a major issue from both the public health and the environmental health perspectives (Huanget al. 2016; Ung-Duck et al. 2016; Huangetal. 2015). Therefore arsenic contamination in drinking water has now become a global issue and is present all over the world (Zheng et al. 2015). Arsenic is widely distributed in nature (in air, water and soil) in the form of either metalloids or chemical compounds. It is used commercially, in pesticide, wood preservative, in the manufacture of glass, paper and semiconductors. Rank wise; it is $20^{\text {th }}$ element in abundance on earth's crust, $14^{\text {th }}$ in seawater and $12^{\text {th }}$ in human body coming from both natural and anthropogenic sources (Rezende et al.
2013;Asadullah et al. 2011; Steven et al.2012; Vinod et al. 2012).As per toxicological studies, organic arsenic was declared to be less toxic in comparison to inorganic arsenic. In general, it was found that organic arsenicals were more rapidly excreted than inorganic forms and pentavalent arsenicals were observed to be cleared faster than trivalent ones (Wang et al. 2012; Spayd et al. 2012; Okkenhaug et al. 2012).

In drinking water, arsenic is found as inorganic and poses a great hazardous effect to human health. Clinical manifestations of arsenic poisoning begin with various forms of cancers including skin; bladder, lung, kidney, liver and prostate cancers. The cardiovascular and neurological effects were also attributed to inorganic arsenic (Chowdhury et al. 2015; Hossain et al. 2014; Eleni et al. 2013; Sinha et al. 2013; Douillet et al. 2013; Zivin et al. 2013).The 
contamination of water from arsenic and its health impact on human have already been reported from 23 regions in different parts of the world including Argentina, Mexico, Mongolia, Germany, Thailand, China, USA, Canada, Hungary, Romania and Vietnam (Flanagan et al. 2012; Ioannis and Athanasios 2006; Kamala et al. 2010; Yanget al.2015; Nguyenet al. 2012; Thiet al.2009; Stangeret al. 2005).

Pakistan is also facing serious public health disasters due to arsenic contaminated water and has acknowledged the need of apprizing drinking water quality and arsenic problem. Different areas of our country have high arsenic concentration in drinking water including ground and surface water (Muhammad Qasim and Mushtaque Ali2017; Fakir et al. 2016; Seema et al. 2016; Sardar et al. 2015; Atta et al. 2016; Sadia et al. 2015; Toqeer et al. 2015; Abbas et al. 2013; Khan et al. 2013;Jakhrani et al. 2011;Baiget al. 2010).

Therefore, the aim of our present study was to evaluate the concentration level of arsenic and other toxic elements in groundwater of Sobhodero and its surroundings with special emphasis to arsenic contamination possibly coming through drinking water sources because in the study area analysis of arsenic concentration in drinking water was not carried out so far, by any government organization or other national agency.

\section{MATERIALS AND METHODS \\ 2.1 Study Area}

Sobhodero District Khairpur is lying between $27^{\circ}$ $32^{\prime}-73^{\circ} 40$ north latitudes and $68^{\circ} 37^{\prime} 19^{\circ} 32^{\prime}$ east longitudes. The study area of present research work is Sobhodero Taluka District Khairpur Mir's which is an agricultural and fertile land and is comingin region of cotton belt of the province of Sindh, Pakistan. TalukaSobhodero comprises nine Union Councils (UCs) namely, Sobhodero, Ranipur, Hingorja,Madd, Sami, Saghyoon,Pirhiyat Shah,Rasoolabad and Gadhiji. The area is covered almost with rural population settled in villages, some small cities with good populationare also available such as Ranipur, Hingorja and Sobhedero itself. The study area is located at the northern part of Sindh province of Pakistan as shown in Figure-1. Moreover, study area is a subtropical region, mostly cold in winter and hot in the summer. The range of temperature is 4 to $46{ }^{\circ} \mathrm{C}$ having more than $230 \mathrm{~mm}$ average rainfall (Shrestha et al. 2002).

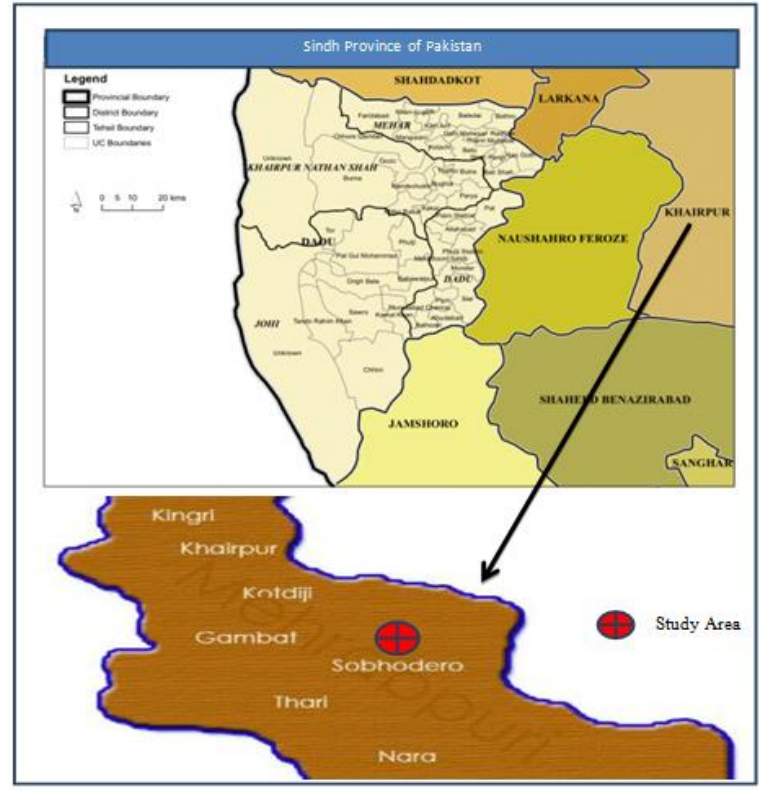

Figure 1: Map of Sindh, Pakistan, Showing study area.

\subsection{Collection and Pretreatment of water Samples}

Three hundred and thirty three (333) groundwater samples were collected form Sobhodero Taluka District Kahirpur on the basis of the Union Councils from various sampling points. The samples were taken in $500 \mathrm{ml}$ polyethylene plastic bottles. Cluster sampling protocol was adopted throughout the work. Samples were collected from tube well and hand pumps by applying below mentioned procedure. After filling water samples in $500 \mathrm{ml}$ plastic (polyethylene) bottles, the bottles were marked with waterproof labels and dully coded for identification. The pre-treatment of the samples was performed as described in paper (Muhammad Qasim and Mushtaque Jakhrani 2017). The pretreated samples were then preserved by adding $10 \% \mathrm{HNO}_{3}$ to bring the $\mathrm{pH}$ of samples less than 2.0. For samples having neutral $\mathrm{pH}$, approximately, $2.5 \mathrm{ml}$ of $10 \% \mathrm{HNO}_{3}$ per 0.5 litter was added. The preserved samples were stored at $0-4{ }^{\circ} \mathrm{C}$ for a minimum period of 48 hours prior to analysis.

\subsection{Reagents and Glassware}

Double de-ionized ultrapure water was used thorough out the research work. Analytical reagent grade $\mathrm{HNO}_{3}$ and $\mathrm{HCl}$, by Merck (Darmstadt, Germany) were used. Pure Argon (99.99\%) gas was used as sheath/carrier gas for atomizer. For the preparation of sodium tetra hydro borate $\left(\mathrm{NaBH}_{4}\right)$ solution, powdered $\mathrm{NaBH}_{4}$ was dissolved in $0.5 \mathrm{M}$ potassium iodide (KI). All the standards for analysis of $\mathrm{As}, \mathrm{Cu}$, 
$\mathrm{Fe}, \mathrm{Ni}, \mathrm{Pb}$ and $\mathrm{Zn}$ were made by dilution method from stock standard $\left(1000 \mathrm{mgL}^{-1}\right)$ solutions.

\subsection{Analysis of Water Samples}

All tube well (TW) and hand pump (HP) water samples collected from different sites were filtered through $0.45 \mu \mathrm{m}$ filter paper. After filtration process, the samples were placed in deep freezer at the temperature of $4^{\circ} \mathrm{C}$ for further analysis. Analysis in respect of $\mathrm{Cu}, \mathrm{Fe}, \mathrm{Ni}, \mathrm{Pb}$ and $\mathrm{Zn}$ was carried out by using Atomic Absorption Spectroscopic technique AAS-100 Analyst by Perkin Elmer. However, As analysis was performed by using AAS coupled with Mercury Hydride Generation System (MHS15) at the Institute of Chemistry, Shah Abdul Latif University, Khairpur, Sindh, Pakistan. Temperature and $\mathrm{pH}$ of water samples were measured by using thermometer and portable $\mathrm{pH}$ meter (781-pH meter Metrohm) respectively in the field.

\subsection{Statistical Analysis}

Results were statistically analyzed for mean value. All results were taken in triplicate manner and reported only mean of the triplicate values. Minitab version 13 software was used along with MS XP Office 2010 version. For correlation among sampling sites and interpreted elements, Pearson correlation SPSS package was used.

\section{Results and discussion}

For most convenient description, groundwater samples were divided into two categories such as hand pump (HP) and tube well (TW) samples. The depth of hand pump samples (HP, $n=243$ ) was varying from 35 to 40 feet and the depth of tube-well samples (TW, $n=90$ ) was varying from 80 to 100 feet. The $\mathrm{pH}$ is one of the most important parameters to test the water quality and it is also a useful test for interpretation of water chemistry. Hence the $\mathrm{pH}$ of both hand pump and tube-well water samples were found neutral and it was within the WHO recommended values (6.5-8.5). The levels of $\mathrm{As}, \mathrm{Cu}, \mathrm{Fe}, \mathrm{Ni}, \mathrm{Pb}$ and $\mathrm{Zn}$ in the study area were tabulated in Tables 1-3.

It was found that level of arsenic was reached up to $58 \mathrm{gL}^{-1}$ in Union Council Madd in sample number 122c. The obtained analysis data indicated that level of As was observed high in both HP samples and TW samples while levels of $\mathrm{Cu}, \mathrm{Ni}$ and $\mathrm{Pb}$ in water samples were found within the safe limits as proposed by WHO. The levels of $\mathrm{Fe}$ and $\mathrm{Zn}$ were found to be slightly higher than WHO permissible limits in HP and TW samples. The results of Fe and $\mathrm{Zn}$ were observed in the range of $20-412 \mu \mathrm{gL}^{-1}$ and $15-420 \mu \mathrm{gL}^{-1}$ respectively, in HP samples, whereas09$100 \mu \mathrm{gL}^{-1}$ and $01-11 \mu \mathrm{gL}^{-1}$ respectively, in TW samples. This type of work has been reported by (Muhammad Qasim and MushtaqueJakhrani2017).

Table.1.Groundwater analysis data ofdifferent Union Councils of Sobhodero, Khairpur, Sindh, Pakistan

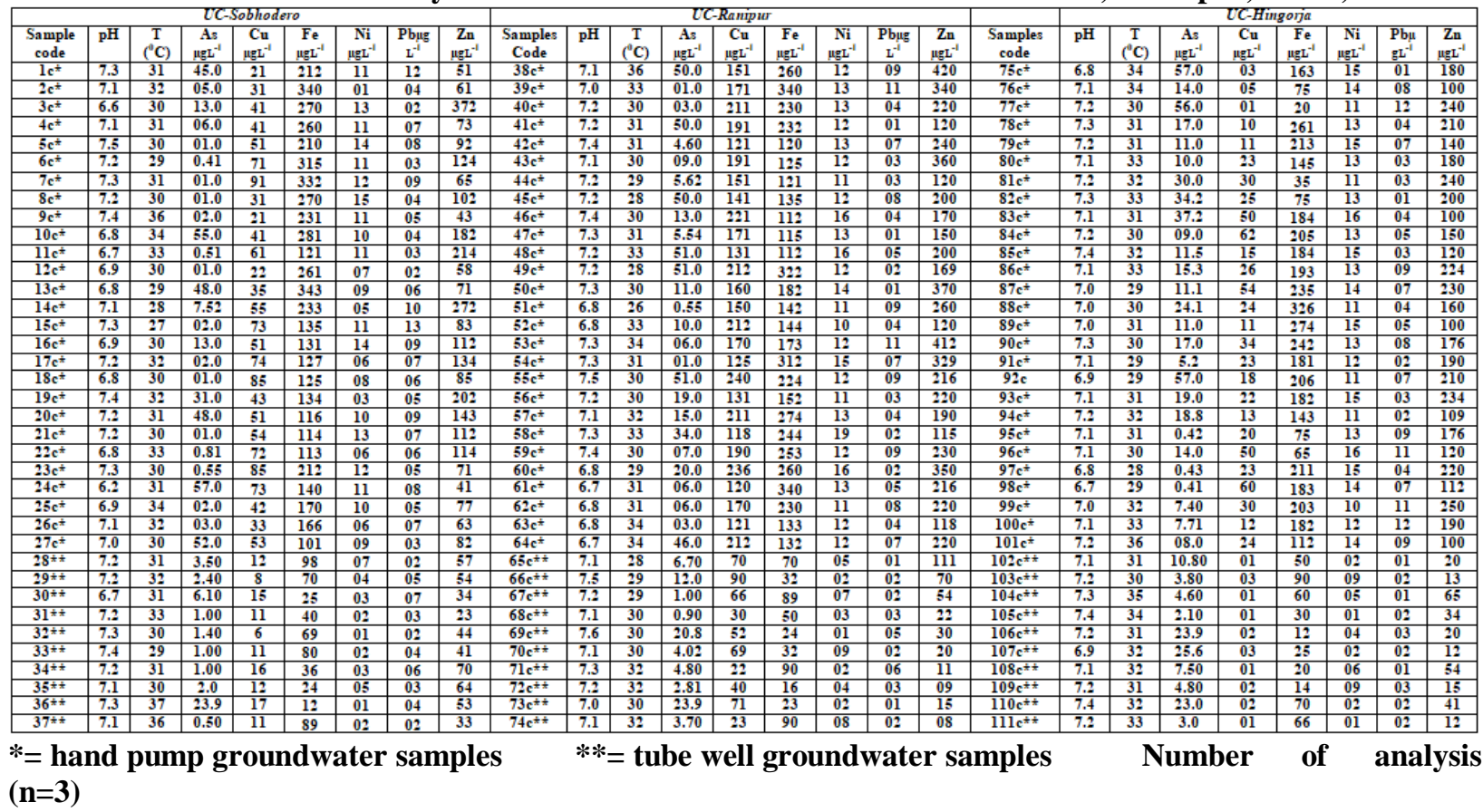


Table.2.Groundwater analysis data of different Union Councils of Sobhodero, Khairpur, Sindh, Pakistan

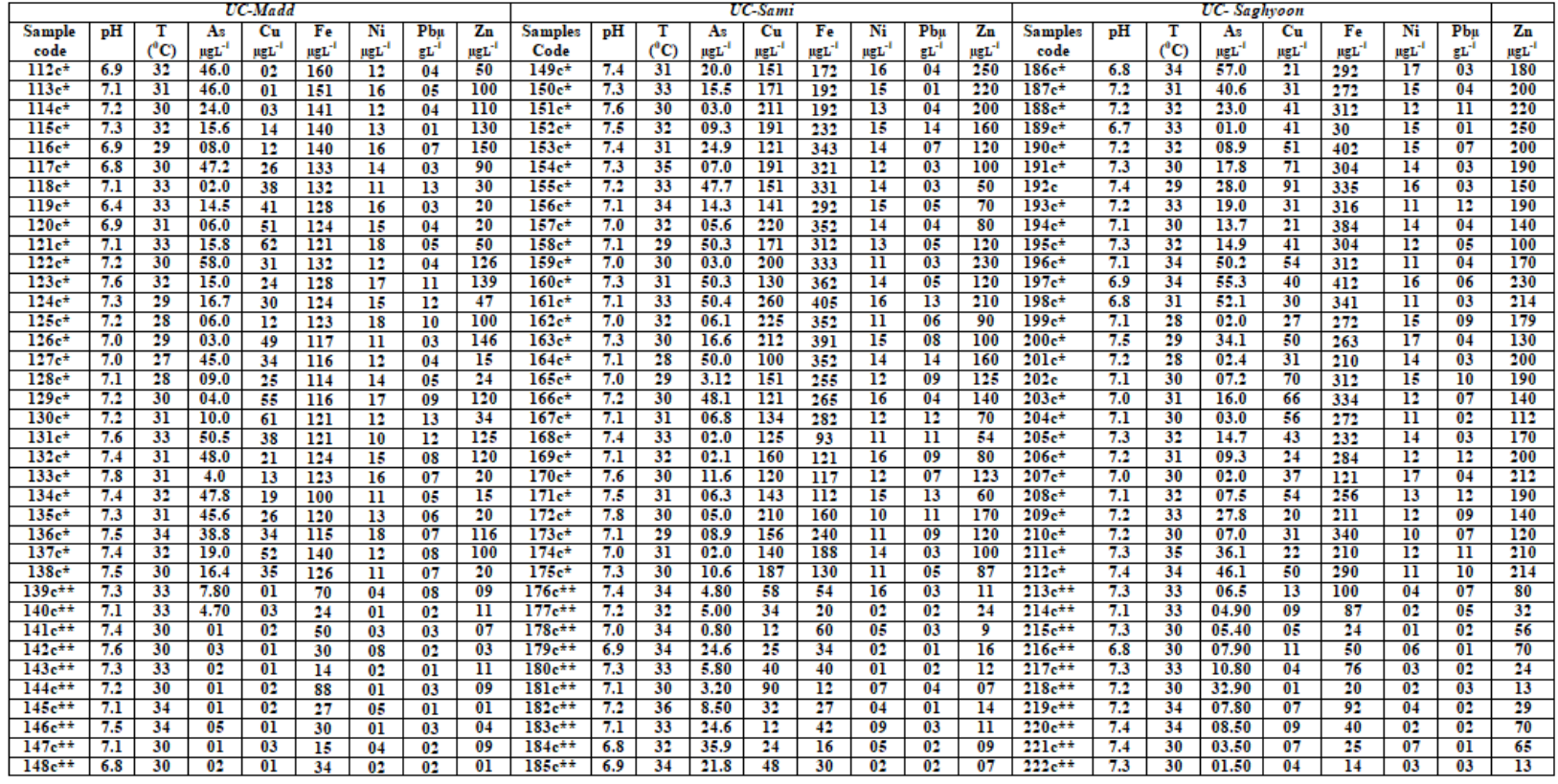

*= hand pump groundwater samples $\quad * *=$ tube well groundwater samples $\quad$ Number of analysis $(\mathbf{n}=3)$

Table.3.Groundwater analysis data of different Union Councils of Sobhodero, Khairpur, Sindh, Pakistan

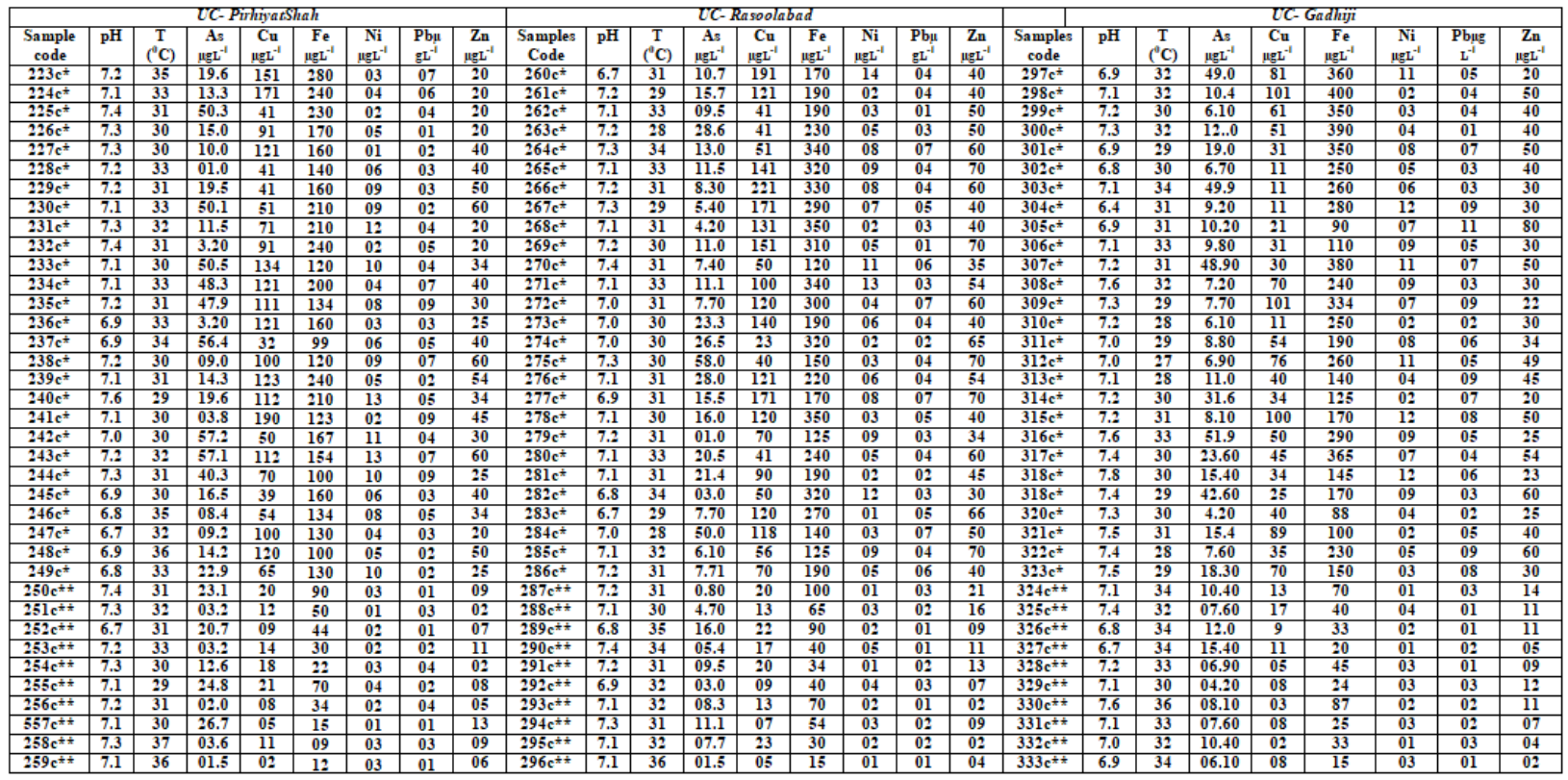

*= hand pump groundwater samples analysis $(\mathbf{n}=3)$
$* *=$ tube well groundwater samples Number

of 
Table.4. Statistical Percentage of Arsenic in groundwater samples of different Union Councils of Sobhodero, Khairpur, Sindh, Pakistan

\begin{tabular}{|c|c|c|c|c|c|c|c|}
\hline $\begin{array}{l}\text { Sr. } \\
\text { No. }\end{array}$ & Sampling Sites & $\begin{array}{c}\text { \% of samples } \\
\text { contaminated } \\
\text { with As }\end{array}$ & $\begin{array}{l}\% \text { of } \\
\text { samples } \\
\text { contaminat } \\
\text { ed with } \mathrm{Cu}\end{array}$ & $\begin{array}{c}\% \text { of } \\
\text { samples } \\
\text { contaminat } \\
\text { ed with Fe }\end{array}$ & $\begin{array}{c}\% \text { of } \\
\text { samples } \\
\text { contaminat } \\
\text { ed with } \mathrm{Ni}\end{array}$ & $\begin{array}{c}\% \text { of } \\
\text { samples } \\
\text { contaminat } \\
\text { ed with } \mathrm{Pb}\end{array}$ & $\begin{array}{c}\% \text { of } \\
\text { samples } \\
\text { contaminat } \\
\text { ed with } \mathrm{Zn}\end{array}$ \\
\hline \multirow[t]{3}{*}{1.} & UC-Sobhodero & & & & & & \\
\hline & $\begin{array}{l}\text { Hand pump water } \\
\mathrm{n}=27\end{array}$ & 33.3 & - & 3.7 & - & - & 3.7 \\
\hline & Tube well water $n=10$ & 10.0 & - & - & - & - & - \\
\hline \multirow[t]{3}{*}{2.} & UC-Ranipur & & & & & & \\
\hline & $\begin{array}{l}\text { Hand pump water } \\
\mathrm{n}=27\end{array}$ & 37.0 & - & 14.8 & - & - & 25.9 \\
\hline & Tube well water $n=10$ & 20.0 & - & - & - & - & - \\
\hline \multirow[t]{3}{*}{3.} & UC-Hingorja & & & & & & \\
\hline & $\begin{array}{l}\text { Hand pump water } \\
n=27\end{array}$ & 66.6 & - & 3,7 & - & - & - \\
\hline & Tube well water $n=12$ & 40.0 & - & - & - & - & - \\
\hline \multirow[t]{3}{*}{4.} & UC-Madd & & & & & & \\
\hline & $\begin{array}{l}\text { Hand pump water } \\
\mathrm{n}=27\end{array}$ & 66.6 & - & - & - & - & - \\
\hline & Tube well water $n=10$ & - & - & - & - & - & - \\
\hline \multirow[t]{3}{*}{5.} & UC-Sami & & & & & & \\
\hline & $\begin{array}{l}\text { Hand pump water } \\
\mathrm{n}=27\end{array}$ & 48.1 & - & 40.7 & - & - & - \\
\hline & Tube well water $n=10$ & 40.0 & - & - & - & - & - \\
\hline \multirow[t]{3}{*}{6.} & UC-Saghyoon & & & & & & \\
\hline & $\begin{array}{l}\text { Hand pump water } \\
\mathrm{n}=\mathbf{2 7}\end{array}$ & 59.2 & - & 48.1 & - & - & - \\
\hline & Tube well water $n=10$ & 20.0 & - & - & - & - & - \\
\hline \multirow[t]{3}{*}{7.} & UC-Pirhiyat shah & & & & & & \\
\hline & $\begin{array}{l}\text { Hand pump water } \\
\mathrm{n}=\mathbf{2 7}\end{array}$ & 70.4 & - & - & - & - & - \\
\hline & Tube well water $n=10$ & 50.0 & - & - & - & - & - \\
\hline \multirow[t]{3}{*}{8.} & UC-Rasoolabad & & & & & & \\
\hline & $\begin{array}{l}\text { Hand pump water } \\
\mathrm{n}=\mathbf{2 7}\end{array}$ & 55.5 & - & 37 & - & - & - \\
\hline & Tube well water $n=05$ & 20.0 & - & - & - & - & - \\
\hline \multirow[t]{3}{*}{9.} & UC-Gadhïi & & & & & & \\
\hline & $\begin{array}{l}\text { Hand pump water } \\
\mathrm{n}=27\end{array}$ & 55.5 & - & 29.6 & - & - & - \\
\hline & Tube well water $n=05$ & 40.0 & - & - & - & - & - \\
\hline
\end{tabular}


Table-5.Temperature, pH and toxic elements ranges in groundwater samples of Sobhodero, Khairpur, Pakistan

\begin{tabular}{|c|c|c|c|c|c|c|c|c|c|c|c|c|c|c|c|c|c|c|c|}
\hline & & \multicolumn{6}{|c|}{ pH } & \multicolumn{6}{|c|}{$\mathrm{T}\left({ }^{0} \mathrm{C}\right)$} & \multicolumn{6}{|c|}{ As } \\
\hline & & \multicolumn{3}{|c|}{ Hand pump } & \multicolumn{3}{|c|}{ Tube well } & \multicolumn{3}{|c|}{ Hand pump } & \multicolumn{3}{|c|}{ Tube well } & \multicolumn{3}{|c|}{ Hand pump } & \multicolumn{3}{|c|}{ Tube well } \\
\hline & & $\begin{array}{l}M \\
\text { in }\end{array}$ & $\begin{array}{l}M \\
a x\end{array}$ & $\begin{array}{c}\text { Me } \\
\text { an }\end{array}$ & $\begin{array}{c}M \\
\text { in }\end{array}$ & $\begin{array}{l}M \\
a .\end{array}$ & $\begin{array}{c}M e \\
a n\end{array}$ & $\begin{array}{l}M \\
\text { in }\end{array}$ & $\begin{array}{l}M \\
a x\end{array}$ & $\begin{array}{l}\text { Me } \\
\text { an }\end{array}$ & $\begin{array}{l}M \\
\text { in }\end{array}$ & $\begin{array}{l}M \\
a x\end{array}$ & $\begin{array}{c}M e \\
a n\end{array}$ & $\begin{array}{l}M \\
\text { in }\end{array}$ & $\begin{array}{l}M \\
a x\end{array}$ & $\mathrm{Mea}$ & $\begin{array}{l}M \\
i \\
n\end{array}$ & $\begin{array}{l}M \\
a x\end{array}$ & $\begin{array}{c}\text { Me } \\
\text { an }\end{array}$ \\
\hline & WHO & \multicolumn{6}{|c|}{$(6.5-8.5)$} & \multicolumn{6}{|c|}{$\left(25-39^{0} \mathrm{C}\right)$} & \multicolumn{6}{|c|}{$10 \mu \mathrm{gL}^{-1}$} \\
\hline $\begin{array}{l}1 \\
.\end{array}$ & $\begin{array}{l}U C- \\
\text { Sobho } \\
\text { dero }\end{array}$ & $\begin{array}{l}6 . \\
6\end{array}$ & $\begin{array}{l}7 . \\
5\end{array}$ & 7.0 & $\begin{array}{l}6 . \\
7\end{array}$ & $\begin{array}{l}7 \\
4\end{array}$ & 7.2 & 27 & 36 & 31 & 29 & 37 & 32 & $\begin{array}{l}0 . \\
41\end{array}$ & $\begin{array}{l}57 \\
.0\end{array}$ & $\begin{array}{l}14 . \\
8\end{array}$ & 0.4 & $\begin{array}{l}23 \\
.9\end{array}$ & $\begin{array}{l}4.2 \\
0\end{array}$ \\
\hline 2 & $\begin{array}{l}\text { UC- } \\
\text { Ranip } \\
\text { ur }\end{array}$ & $\begin{array}{l}6 . \\
7\end{array}$ & $\begin{array}{l}7 . \\
5\end{array}$ & 7.1 & $\begin{array}{l}7 . \\
0\end{array}$ & $\begin{array}{l}7 \\
6\end{array}$ & 7.2 & 26 & 36 & 31 & 28 & 32 & 30 & $\begin{array}{l}0 . \\
6\end{array}$ & $\begin{array}{l}51 \\
.0\end{array}$ & $\begin{array}{l}19 . \\
6\end{array}$ & 0.9 & $\begin{array}{l}23 \\
.9\end{array}$ & $\begin{array}{l}8.1 \\
0\end{array}$ \\
\hline $\begin{array}{l}3 \\
.\end{array}$ & $\begin{array}{l}\text { UC- } \\
\text { Hingor } \\
\text { ja }\end{array}$ & $\begin{array}{l}6 . \\
7\end{array}$ & $\begin{array}{l}7 . \\
4\end{array}$ & 7.1 & $\begin{array}{l}6 . \\
9\end{array}$ & $\begin{array}{l}7 \\
4\end{array}$ & 7.2 & 28 & 36 & 31 & 30 & 35 & 32 & $\begin{array}{l}0 . \\
4\end{array}$ & $\begin{array}{l}57 \\
.0\end{array}$ & $\begin{array}{l}18 . \\
7\end{array}$ & 2.1 & $\begin{array}{l}25 \\
.6\end{array}$ & $\begin{array}{l}10 . \\
9\end{array}$ \\
\hline 4 & $\begin{array}{l}\text { UC- } \\
\text { Madd }\end{array}$ & $\begin{array}{l}6 . \\
9\end{array}$ & $\begin{array}{l}7 . \\
8\end{array}$ & 7.2 & $\begin{array}{l}6 . \\
8\end{array}$ & $\begin{array}{l}7 \\
6\end{array}$ & 6.8 & 27 & 34 & 31 & 30 & 34 & 32 & $\begin{array}{l}2 . \\
0\end{array}$ & $\begin{array}{l}58 \\
.0\end{array}$ & $\begin{array}{l}24 . \\
5\end{array}$ & 0.4 & $\begin{array}{l}08 \\
.6\end{array}$ & $\begin{array}{l}3.9 \\
0\end{array}$ \\
\hline $\begin{array}{l}5 \\
.\end{array}$ & $\begin{array}{l}\text { UC- } \\
\text { Sami }\end{array}$ & $\begin{array}{l}7 . \\
0\end{array}$ & $\begin{array}{l}7 . \\
8\end{array}$ & 7.3 & $\begin{array}{l}6 . \\
8\end{array}$ & $\begin{array}{l}7 \\
4 \\
\end{array}$ & 7.1 & 28 & 35 & 31 & 30 & 36 & 33 & $\begin{array}{l}2 . \\
0\end{array}$ & $\begin{array}{l}50 \\
.4\end{array}$ & $\begin{array}{l}17 . \\
8\end{array}$ & 0.8 & $\begin{array}{l}35 \\
.9\end{array}$ & $\begin{array}{l}13 . \\
5\end{array}$ \\
\hline $\begin{array}{l}6 \\
\cdot\end{array}$ & $\begin{array}{l}U C- \\
\text { Saghy } \\
\text { oon }\end{array}$ & $\begin{array}{l}6 . \\
7\end{array}$ & $\begin{array}{l}7 . \\
5\end{array}$ & 7.2 & $\begin{array}{l}6 . \\
8\end{array}$ & $\begin{array}{l}7 \\
3\end{array}$ & 7.2 & 28 & 35 & 31 & 30 & 34 & 32 & $\begin{array}{l}1 . \\
0\end{array}$ & $\begin{array}{l}55 \\
.3\end{array}$ & $\begin{array}{l}20 . \\
5\end{array}$ & 1.5 & $\begin{array}{c}32 \\
.9\end{array}$ & $\begin{array}{l}09 . \\
0\end{array}$ \\
\hline $\begin{array}{l}7 \\
.\end{array}$ & $\begin{array}{l}\text { UC- } \\
\text { Pirhiy } \\
\text { at } \\
\text { shah }\end{array}$ & $\begin{array}{l}6 . \\
7\end{array}$ & $\begin{array}{l}7 . \\
6\end{array}$ & 7.1 & $\begin{array}{l}6 . \\
7\end{array}$ & $\begin{array}{l}7 \\
4\end{array}$ & 6.7 & 29 & 36 & 32 & 29 & 37 & 32 & $\begin{array}{l}1 . \\
0\end{array}$ & $\begin{array}{l}57 \\
.2\end{array}$ & $\begin{array}{l}24 . \\
9\end{array}$ & 1.6 & $\begin{array}{l}26 \\
.8\end{array}$ & $\begin{array}{l}12 . \\
4\end{array}$ \\
\hline $\begin{array}{l}8 \\
.\end{array}$ & $\begin{array}{l}\text { UC- } \\
\text { Rasool } \\
\text { abad }\end{array}$ & $\begin{array}{l}6 . \\
7\end{array}$ & $\begin{array}{l}7 . \\
4\end{array}$ & 7.1 & $\begin{array}{l}6 . \\
8\end{array}$ & $\begin{array}{l}7 \\
5\end{array}$ & 7.2 & 28 & 34 & 31 & 30 & 35 & 32 & $\begin{array}{l}1 . \\
0\end{array}$ & $\begin{array}{l}58 \\
.0\end{array}$ & $\begin{array}{l}15 . \\
9\end{array}$ & 0.8 & $\begin{array}{l}16 \\
.0\end{array}$ & $\begin{array}{l}7.1 \\
0\end{array}$ \\
\hline \multirow[t]{3}{*}{$\begin{array}{l}9 \\
\text {. }\end{array}$} & $\begin{array}{l}U C- \\
\text { Gadhij } \\
i\end{array}$ & $\begin{array}{l}6 . \\
4\end{array}$ & $\begin{array}{l}7 . \\
8\end{array}$ & 7.2 & $\begin{array}{l}6 . \\
7\end{array}$ & $\begin{array}{l}7 \\
6\end{array}$ & 7.1 & 27 & 34 & 30 & 30 & 36 & 33 & $\begin{array}{l}4 . \\
2\end{array}$ & $\begin{array}{l}51 \\
.9\end{array}$ & $\begin{array}{l}18 . \\
7\end{array}$ & 4.2 & $\begin{array}{l}15 \\
.4\end{array}$ & $\begin{array}{l}8.9 \\
0\end{array}$ \\
\hline & & \multicolumn{6}{|c|}{$\mathrm{Cu}$} & \multicolumn{6}{|c|}{ Fe } & \multicolumn{6}{|c|}{$\mathbf{P b}$} \\
\hline & WHO & \multicolumn{6}{|c|}{$2000 \mu \mathrm{gL}^{-1}$} & \multicolumn{6}{|c|}{$300 \mu \mathrm{gL}^{-1}$} & \multicolumn{6}{|c|}{$100 \mu \mathrm{gL}^{-1}$} \\
\hline $\begin{array}{l}1 \\
.\end{array}$ & $\begin{array}{l}\text { UC- } \\
\text { Sobho } \\
\text { dero }\end{array}$ & 21 & 91 & 52 & 06 & 1 & 12 & $\begin{array}{c}10 \\
1\end{array}$ & $\begin{array}{c}34 \\
3\end{array}$ & $\begin{array}{c}20 \\
2\end{array}$ & 12 & 98 & 54 & 02 & 13 & 06 & 02 & 07 & 04 \\
\hline $\begin{array}{l}2 \\
\text {. }\end{array}$ & $\begin{array}{l}\text { UC- } \\
\text { Ranip } \\
\text { ur }\end{array}$ & $\begin{array}{c}11 \\
8\end{array}$ & $\begin{array}{c}24 \\
0\end{array}$ & $\begin{array}{c}17 \\
9\end{array}$ & 22 & 9 & $\begin{array}{c}53 . \\
0\end{array}$ & $\begin{array}{c}11 \\
2\end{array}$ & $\begin{array}{c}34 \\
0\end{array}$ & $\begin{array}{c}20 \\
1\end{array}$ & 16 & 90 & 52 & 01 & 11 & 05 & 01 & 06 & $\mathbf{0 3}$ \\
\hline $\begin{array}{l}3 \\
\text {. }\end{array}$ & $\begin{array}{l}U C- \\
\text { Hingor } \\
\text { ja }\end{array}$ & 01 & 62 & $\begin{array}{c}25 . \\
0\end{array}$ & 01 & 0 & 02 & 20 & $\begin{array}{c}32 \\
6\end{array}$ & $\begin{array}{c}16 \\
9\end{array}$ & 12 & 90 & 44 & 01 & 12 & 06 & 01 & 03 & 02 \\
\hline 4 & $\begin{array}{l}\text { UC- } \\
\text { Madd }\end{array}$ & 01 & 62 & 30 & 01 & 0 & 02 & $\begin{array}{c}10 \\
0\end{array}$ & $\begin{array}{c}16 \\
0\end{array}$ & $\begin{array}{c}12 \\
7\end{array}$ & 14 & 88 & 38 & 01 & 13 & 07 & 01 & 08 & 03 \\
\hline 5 & $\begin{array}{l}\text { UC- } \\
\text { Sami }\end{array}$ & $\begin{array}{c}10 \\
0\end{array}$ & $\begin{array}{c}26 \\
0\end{array}$ & $\begin{array}{l}16 \\
6.0\end{array}$ & 12 & 6 & 34 & 93 & $\begin{array}{c}40 \\
5 \\
\end{array}$ & $\begin{array}{c}25 \\
6 \\
\end{array}$ & 12 & 60 & 33 & 01 & 14 & 07 & 01 & 04 & 02 \\
\hline $\begin{array}{l}6 \\
\text {. }\end{array}$ & $\begin{array}{l}U C- \\
\text { Saghy } \\
\text { oon }\end{array}$ & 20 & 91 & 42 & 01 & 1. & 07 & 30 & $\begin{array}{c}41 \\
2\end{array}$ & $\begin{array}{c}28 \\
2\end{array}$ & $\begin{array}{c}14 \\
1\end{array}$ & $\begin{array}{r}10 \\
0\end{array}$ & 53 & 01 & 12 & 06 & 01 & 07 & 03 \\
\hline $\begin{array}{l}7 \\
\text {. }\end{array}$ & $\begin{array}{l}U C- \\
\text { Pirhiy } \\
\text { at }\end{array}$ & 32 & $\begin{array}{c}19 \\
0\end{array}$ & 93 & 02 & 2 & 12 & 99 & $\begin{array}{c}28 \\
0\end{array}$ & $\begin{array}{c}16 \\
8\end{array}$ & 09 & 90 & 38 & 01 & 09 & 05 & 01 & 04 & 2.0 \\
\hline
\end{tabular}




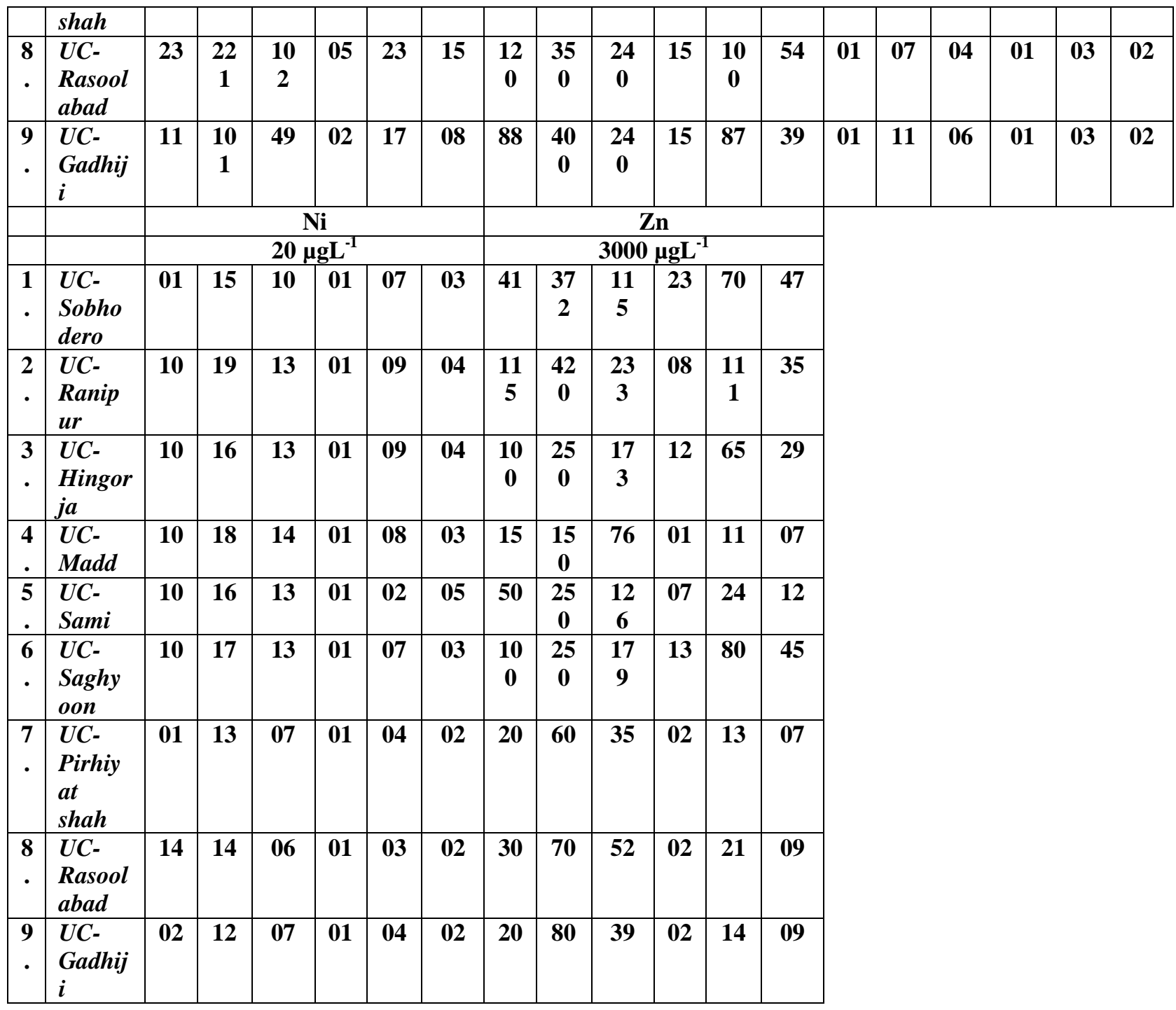

Table.6. Analytical ranges of data of groundwater samples of Sobhodero, Khairpur, Sindh, Pakistan

\begin{tabular}{|c|c|c|c|c|c|c|c|}
\hline & \multirow{2}{*}{$\begin{array}{c}\text { Recommended } \\
\text { values WHO(2010) }\end{array}$} & \multicolumn{3}{|c|}{ Hand pump $n=243^{a}$} & \multicolumn{3}{|c|}{ Tube well $n=90^{a}$} \\
\hline & & Min & $\operatorname{Max}$ & Average & Min & $\operatorname{Max}$ & Average \\
\hline pH & $(6.5-8.5)$ & 6.4 & 7.8 & 7.1 & 6.7 & 7.6 & 7.2 \\
\hline $\mathbf{T}\left({ }^{0} \mathrm{C}\right)$ & $\left(25-39{ }^{0} \mathrm{C}\right)$ & 26 & 36 & 31 & 28 & 37 & 32.0 \\
\hline As $\mu \mathrm{gL}^{-1}$ & $\left(0-10 \mu \mathrm{gL}^{-1}\right)$ & 0.41 & 58.0 & 19.5 & 0.4 & 35.9 & 8.66 \\
\hline $\mathrm{Cu} \mu \mathrm{gL}^{-1}$ & $\left(0-2000 \mu \mathrm{gL}^{-1}\right)$ & 70 & 260 & 85.0 & 01 & 90 & 16.0 \\
\hline Fe $\mu \mathrm{gL}^{-1}$ & $\left(0-300 \mu \mathrm{gL}^{-1}\right)$ & 20 & 412 & 209 & 09 & 100 & 45 \\
\hline $\mathrm{Pb} \mu \mathrm{gL}^{-1}$ & $\left(0-100 \mu g L^{-1}\right)$ & 01 & 14 & 06 & 01 & 08 & $\mathbf{0 3}$ \\
\hline $\mathrm{Ni} \mu \mathrm{gL}^{-1}$ & $\left(0-20 \mu \mathrm{gL}^{-1}\right)$ & 01 & 19 & $\mathbf{1 0 . 6}$ & 01 & 09 & 04 \\
\hline $\operatorname{Zn} \mu g L^{-1}$ & $\left(0-3000 \mu \mathrm{gL}^{-1}\right)$ & 15 & 420 & 114 & 01 & 111 & 22 \\
\hline
\end{tabular}

${ }^{\mathrm{a}}$ No. of samples 
Table.7. Correlation (linear) \& coefficient matrix for As in HP water samples of study area.

\begin{tabular}{|c|c|c|c|c|c|c|c|c|c|c|}
\hline $\begin{array}{l}\text { Sr. } \\
\text { No }\end{array}$ & & $\begin{array}{c}\text { UC- } \\
\text { Sobhod } \\
\text { ero }\end{array}$ & $\begin{array}{c}\text { UC- } \\
\text { Ranip } \\
\text { ur }\end{array}$ & $\begin{array}{c}\text { UC- } \\
\text { Hingorja }\end{array}$ & $\begin{array}{l}\text { UC- } \\
\text { Madd }\end{array}$ & $\begin{array}{l}\text { UC- } \\
\text { Sami }\end{array}$ & $\begin{array}{l}\text { UC- } \\
\text { Saghyo } \\
\text { on }\end{array}$ & $\begin{array}{l}\text { UC-Pirhiyat } \\
\text { shah }\end{array}$ & $\begin{array}{c}\text { UC- } \\
\text { Rasoolaba } \\
\text { d }\end{array}$ & $\begin{array}{c}\text { UC- } \\
\text { Gadhiji }\end{array}$ \\
\hline 1. & $\begin{array}{l}U C- \\
\text { Sobhodero }\end{array}$ & 1 & & & & & & & & \\
\hline 2. & UC-Ranipur & .002 & 1 & & & & & & & \\
\hline 3. & UC-Hingorja & -.036 & .285 & 1 & & & & & & \\
\hline 4. & UC-Madd & .172 & .094 & -.233 & 1 & & & & & \\
\hline 6. & UC-Saghyoon & .285 & .348 & .115 & .172 & .228 & 1 & & & \\
\hline 7. & $\begin{array}{l}\text { UC-Pirhiyat } \\
\text { shah }\end{array}$ & -.058 & .344 & .084 & .077 & -.036 & .355 & 1 & & \\
\hline 8. & $\begin{array}{l}\text { UC- } \\
\text { Rasoolabad }\end{array}$ & -.259 & -.203 & -.154 & .022 & .135 & $-.430 *$ & -.238 & 1 & \\
\hline
\end{tabular}

Table -8. Correlation (linear) \& coefficient matrix for As in HP water samples of study area

\begin{tabular}{|c|c|c|c|c|c|c|}
\hline & As & $\mathbf{C u}$ & $\mathrm{Fe}$ & $\mathbf{N i}$ & $\mathbf{P b}$ & Zn \\
\hline As & 1 & & & & & \\
\hline $\mathbf{C u}$ & .065 & 1 & & & & \\
\hline $\mathbf{F e}$ & $.221^{* * *}$ & $.436^{* * *}$ & 1 & & & \\
\hline $\mathbf{N i}$ & .186 & $.268^{* * *}$ & $.360^{* * *}$ & 1 & & \\
\hline $\mathbf{P b}$ & .103 & $.218^{* * *}$ & $.294^{* *}$ & $.334^{* * *}$ & 1 & \\
\hline $\mathbf{Z n}$ & $.148^{* *}$ & $.370^{* *}$ & $.392^{* * *}$ & $.518^{* *}$ & $.320 * *$ & 1 \\
\hline
\end{tabular}

* Correlation is significant at the 0.05 level $\mathbf{p}<0.05 * *$ Correlation is significant at the 0.01 level $\mathbf{p}<0.01$

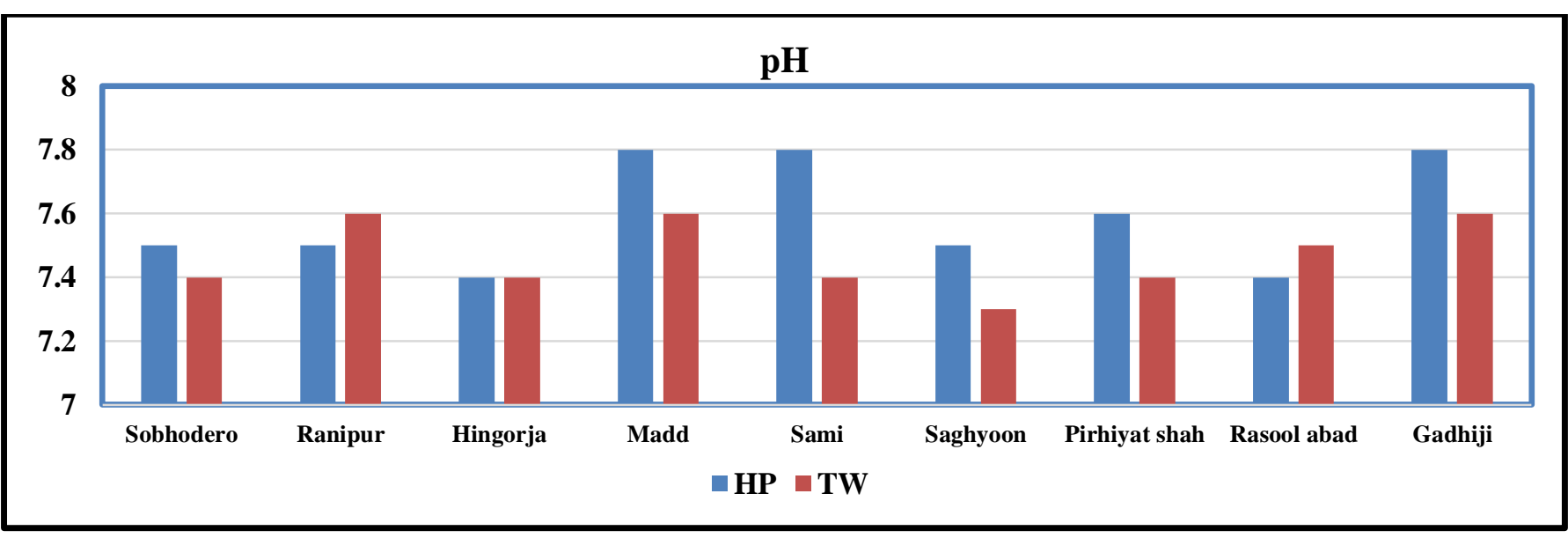

Figure 2Comparison of $\mathrm{pH}$ between HP and TW samples in various Union Councils of study area. 


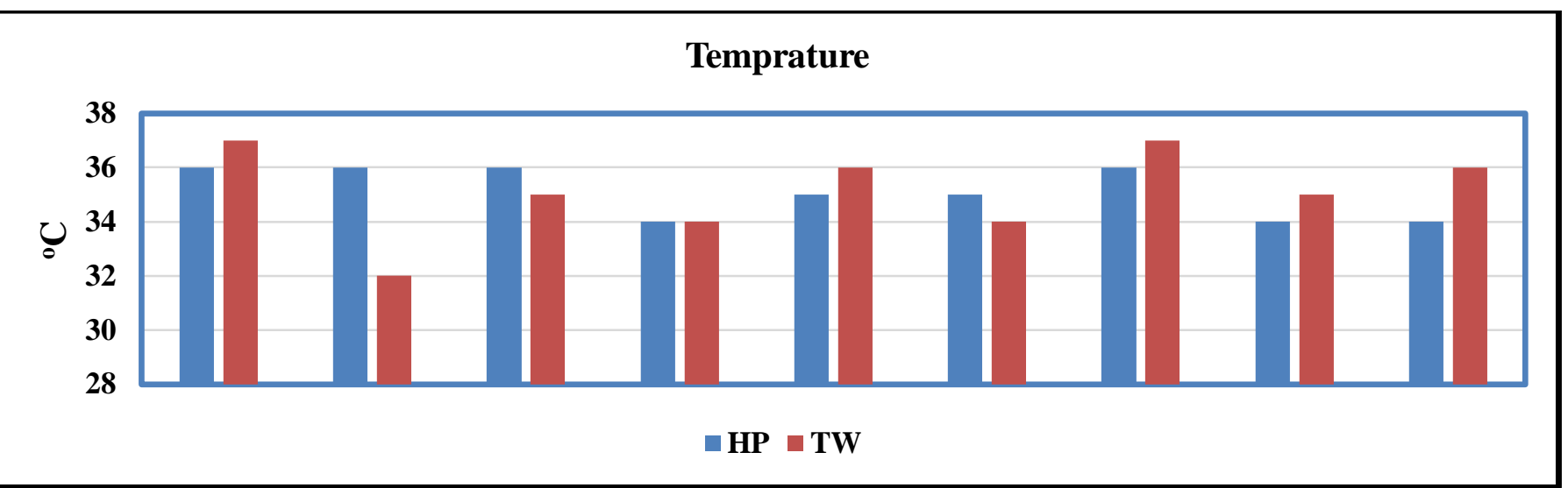

Figure 3 Comparison of temperature between HP and TW samples in various Union Councils of study area.

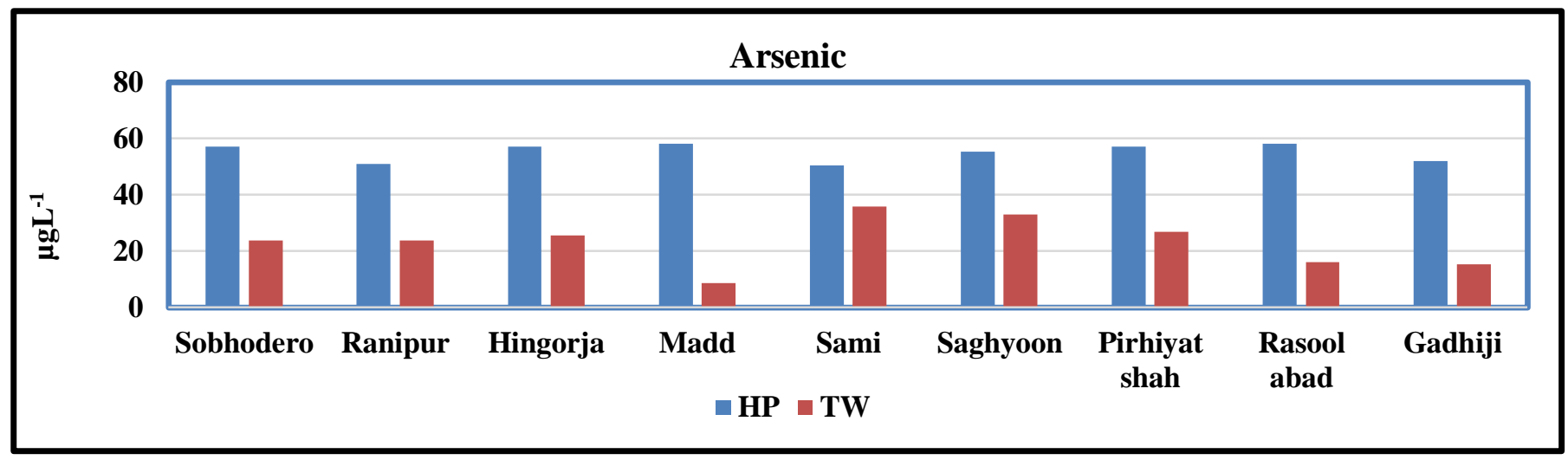

Figure 4 Comparison of arsenic concentration between HP and TW samples in various Union Councils of study area.

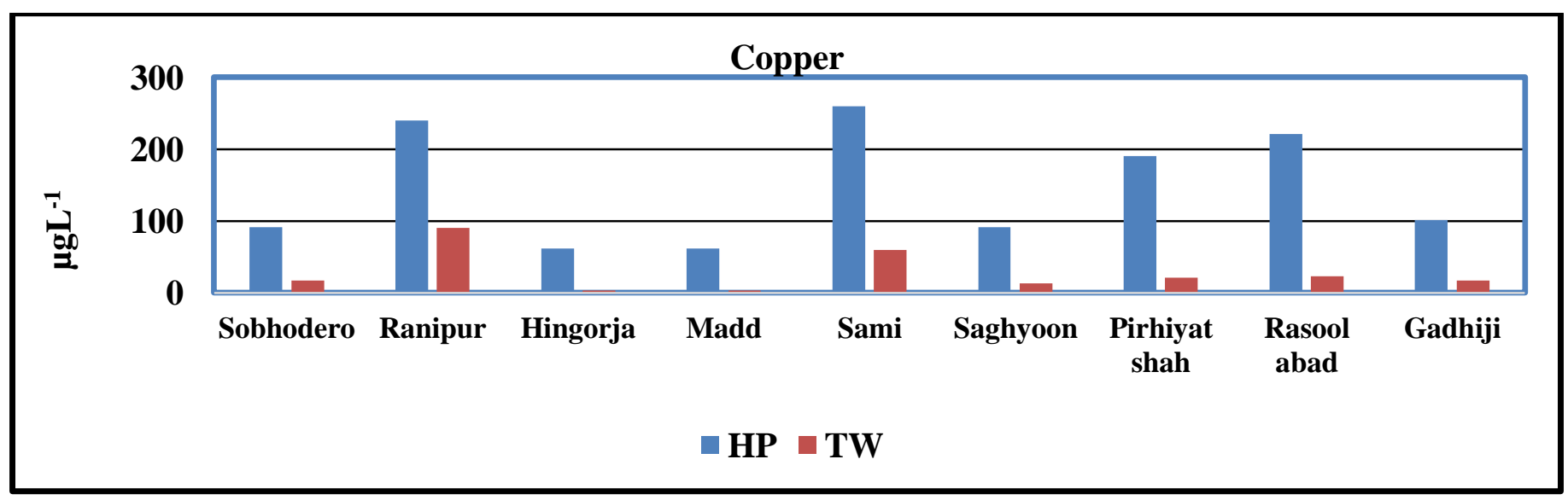

Figure 5 Comparison of copper concentration between HP and TW samples in various Union Councils of study area. 


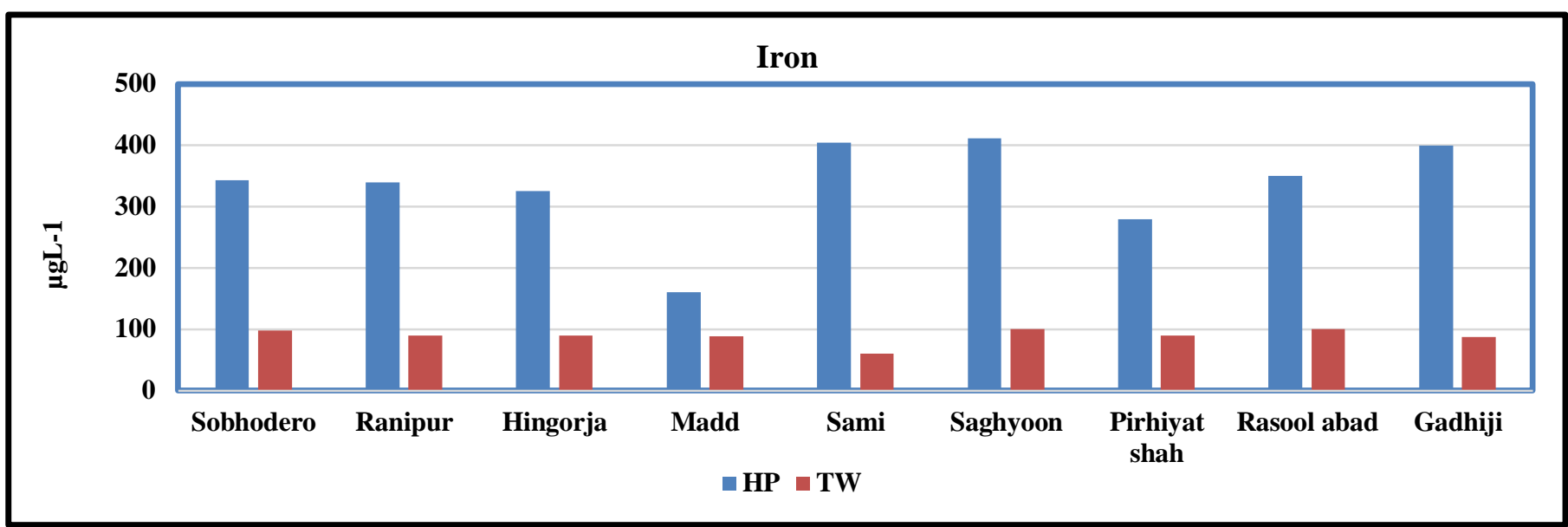

Figure 6 Comparison of iron concentration between HP and TW samples in various Union Councils of study area.

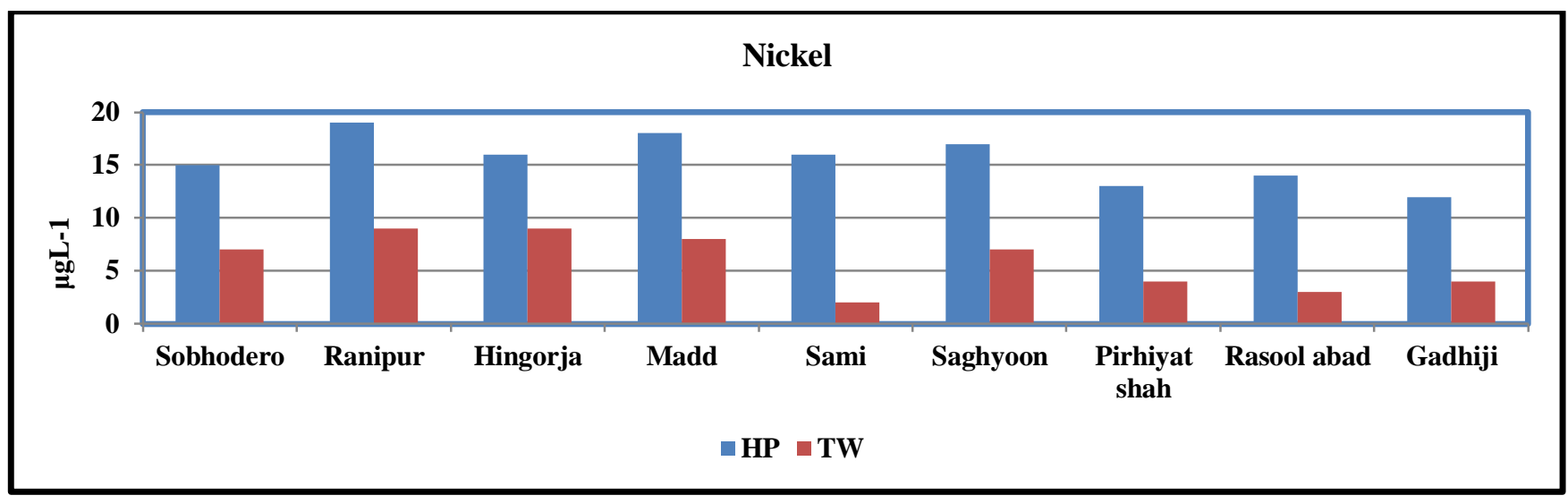

Figure 7 Comparison of nickel concentration between HP and TW samples in various Union Councils of study area.

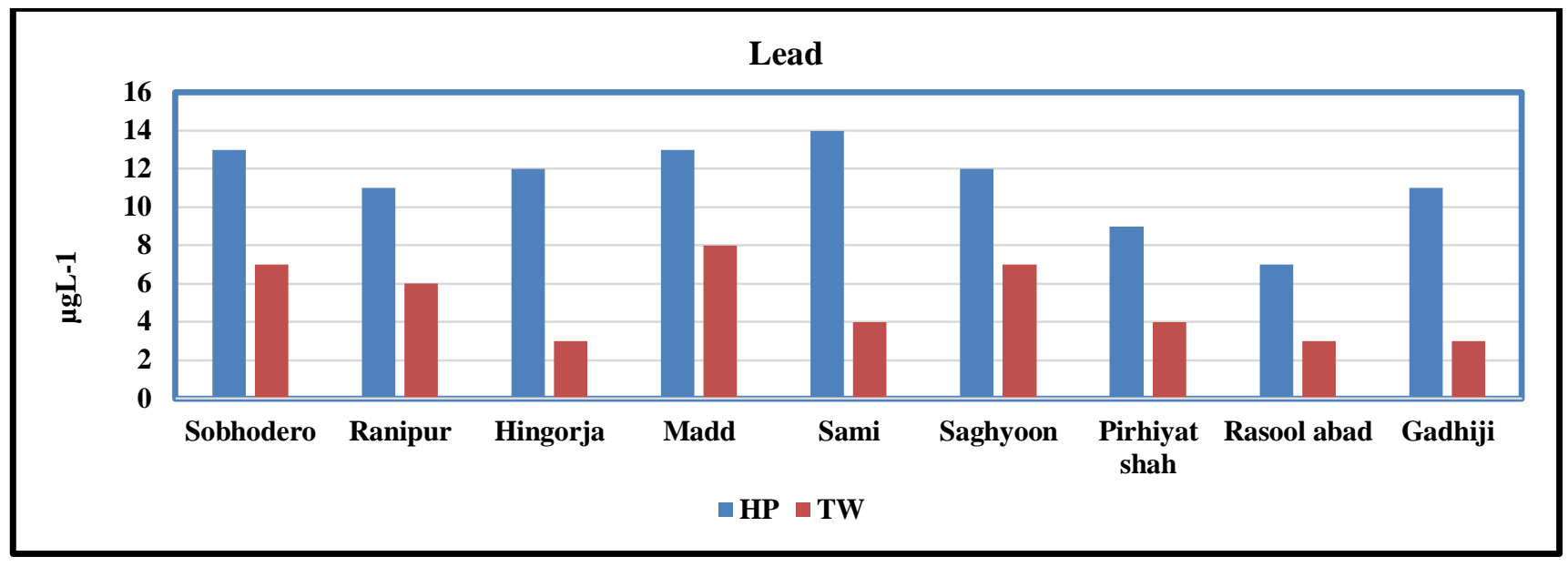

Figure 8 Comparison of lead concentration between HP and TW samples in various Union Councils of study area 


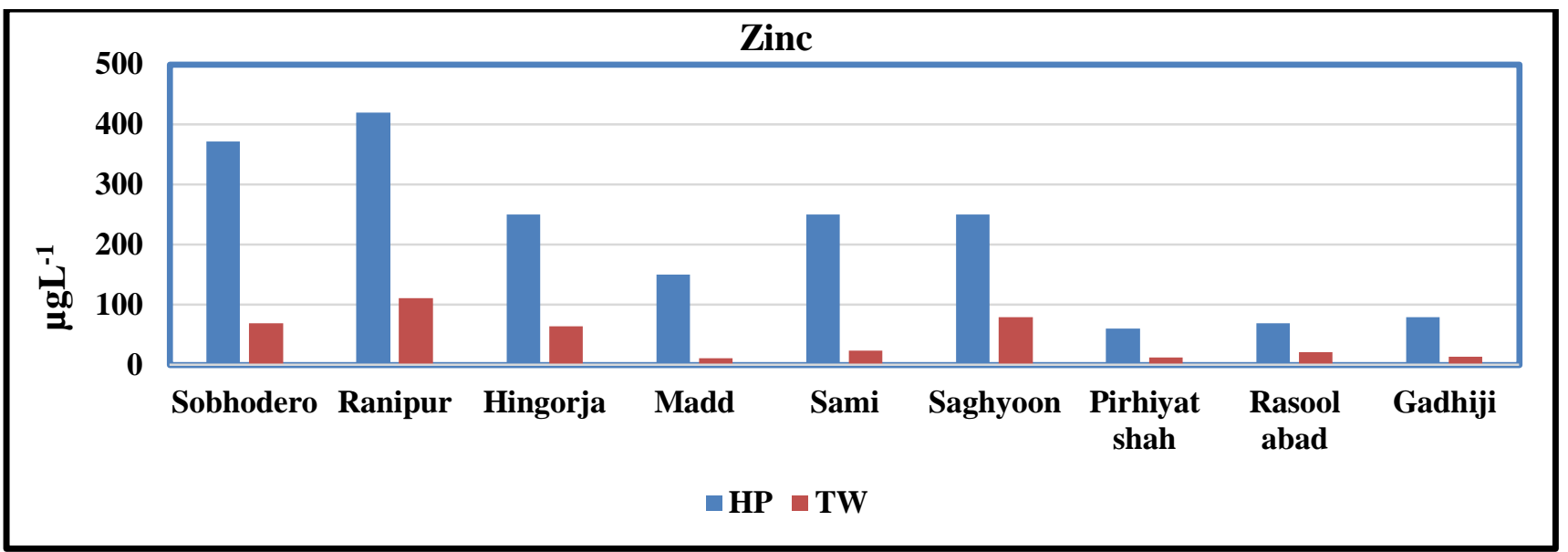

Figure 9 Comparison of zinc concentration between HP and TW samples in various Union Councils of study area.

The percentage of samples contaminated by arsenic and other elements like $\mathrm{Cu}, \mathrm{Fe}, \mathrm{Ni}, \mathrm{Pb}$ and $\mathrm{Zn}$ is given in Table-4. In Union Council Hingorja, arsenic contamination was indicated as $66.6 \%$ in HP and $40 \%$ TW samples. Maximum number of samples examined in this Union Council showed arsenic concentration five times higher than WHO specified limit $\left(10 \mu \mathrm{gL}^{-1}\right)$. The percentage of arsenic contamination at sampling site of Sobhodero, Ranipur, Hingorja, Madd, Sami, Saghyoon, pirhiyat Shah, Rasool Abad and Ghadhi was found as 33.3, $37 \%, 66.6 \%, 66.6 \%, 48.1 \%, 59.2 \%, 70.4 \%, 55.5 \%$ and $55.5 \%$ respectively, in HP samples, whereas for TW samples the respective percentages were observed as $10 \%, 20 \%, 40 \%, 0 \%, 40 \%, 20 \%, 50 \%$, $20 \%$ and $40 \%$ correspondingly. This work is in accordance to the previously reported studies (Mandaland Suzuki 2002; Muhammad Qasim and Mushtaque Ali 2017).

Tables-5was corresponding to statistical results of all parameters of TW and HP samples in minimum and maximum values/concentrations. The levels of $\mathrm{pH}$, temperature, $\mathrm{As}, \mathrm{Cu}, \mathrm{Fe}, \mathrm{Ni}, \mathrm{Pb}$ and $\mathrm{Zn}$ in ranges were found in the range of $6.4-7.8,26-36{ }^{\circ} \mathrm{C}, 0.41$ $58 \mu \mathrm{gL}^{-1}, 70-260 \mu \mathrm{gL}^{-1}, 20-412 \mu \mathrm{gL}^{-1}, 0-19 \mu \mathrm{gL}^{-1}, 1-$ $14 \mu \mathrm{gL}^{-1}$ and $15-420 \mu \mathrm{gL}^{-1}$ respectively in $\mathrm{HP}$ groundwater samples of Sobhodero, but in case TW samples the values were observed as 6.7-7.6, 28-37 ${ }^{0} \mathrm{C}, 0.4-36 \mu \mathrm{gL}^{-1}, 1-90 \mu \mathrm{gL}^{-1}, 9-100 \mu \mathrm{gL}^{-1}, 1-9 \mu \mathrm{gL}^{-1}, 1-$ $8 \mu \mathrm{gL}^{-1}$ and $1-111 \mu \mathrm{gL}^{-1}$ correspondingly. Graphically, the comparative levels of HP and TW samples in respect to $\mathrm{pH}$, temperature, $\mathrm{As}, \mathrm{Cu}, \mathrm{Fe}, \mathrm{Ni}, \mathrm{Pb}$ and $\mathrm{Zn}$ were shown in Figures 2-7.

The concentration of $\mathrm{Fe}$ was found high in $\mathrm{HP}$ samples while least contamination was seen in TW samples of the study area. It was observed that in Union Council Saghyoon, the maximum level of $\mathrm{Fe}$ was found as $412 \mu \mathrm{gL}^{-1}$ in HP sample having code number $197 \mathrm{c} *$ while in UC Madd, the level was observed in safe limit for Fe. The maximum Level of Fe was noted at $405 \mu \mathrm{gL}^{-1}$ in HP sample of UC Sami which was more than WHO permissible limit of $\left(300 \mu \mathrm{gL}^{-1}\right)$. Samples of UC Pirhiyat were found within the safe limits while samples of UCs Gadhiji, Rasoolabad, Sobhedero, Ranipure and Hingorja were found polluted with maximum $\mathrm{Fe}$ concentration as $400 \mu \mathrm{gL}^{-1}, 350 \mu \mathrm{gL}^{-1}, 343 \mu \mathrm{gL}^{-1}, 340 \mu \mathrm{gL}^{-1}, 326 \mu \mathrm{gL}^{-1}$ respectively. Many studies showed that there are various ways for high level of metals in water (Hudak 2000; Finkelman et al. 2002), viz. oxidation of many arsenic ores, volcanoes and use of limitless pesticides (Welch et al. 2000). As per reports researchers, favorable conditions for the uptake of trace and toxic metals in the soil might be provided by the saline environment (Nickson et al. 2005).

The concentration of As was found almost high in HP samples as compared to TW sample in groundwater of the study area as mentioned in Table-6.The maximum concentration of As in HP samples was found as $58 \mu \mathrm{gL}^{-1}, 58 \mu \mathrm{gL}^{-1}, 57.2 \mu \mathrm{gL}^{-1}, 57 \mu \mathrm{gL}^{-1}$, $57 \mu \mathrm{gL}^{-1}, 55.3 \mu \mathrm{gL}^{-1}, 52 \mu \mathrm{gL}^{-1}$ and $50.4 \mu \mathrm{gL}^{-1}$ whereas in TW samples maximum As concentration was found as $8.6 \mu \mathrm{gL}^{-1}, 8.6 \mu \mathrm{gL}^{-1}, 24.0 \mu \mathrm{gL}^{-1}, 26.8 \mu \mathrm{gL}^{-1}, 25.6 \mu \mathrm{gL}^{-}$ ${ }^{1}, 33 \mu \mathrm{gL}^{-1}, 16 \mu \mathrm{gL}^{-1} 24.0 \mu \mathrm{gL}^{-1}$, and $36.0 \mu \mathrm{gL}^{-1}$ in UCs Madd, Rasoolabad, Pirhiyat Shah, Sobhedero, Saghyoon, Gadhiji, Ranipur and Sami respectively. In case of UC Madd, the As concentration was found within safe limit as $8.6 \mu \mathrm{gL}^{-1}$. The observed concentration ranges of As in $\mathrm{HP}\left(19.5-58 \mu \mathrm{gL}^{-1}\right)$ and in TW (8.6-36.0 $\mathrm{gLL}^{-1}$ ) were comparatively less than other countries like Chile and Bangladesh (Sullivan 1969; Find 2001).

The enormous uses of pesticides particularly on cotton crops are responsible for soil and groundwater 
contamination. Uses of fertilizer by un-educated farmer followed by non-scientific method are the major cause of groundwater pollution in the study area. Although there are many other sources of pollution of these toxic heavy metals but it has been observed that domestic waste, pesticides, fertilizer etc might be the major source of heavy metals contamination in underground and surface water (Arain et al. 2007; Wang and Shpeyzer 1997; Mandaland Suzuki 2002).

\subsection{Correlation coefficient $(r)$}

The correlation coefficient $(r)$ indicate the extent of relationship between two variables, one estimates the presence of the other (Sidauruket al.1998). The correlation coefficient among nine union councils for As in groundwater was analyzed and are given inTable-7.The Pearson correlation for different sampling sites indicated significant positive correlation between sampling sites Gadhiji and Ranipur(r=0.392), Saghyoon with Pirhiyat Shah $(\mathrm{r}=0.355)$, while negative correlation was seen between sampling site Saghyoon with Rasoolabad having regression coefficient of $.430 *$ correspondingly.

Correlation study of As with other elements such as $\mathrm{Cu}, \mathrm{Fe}, \mathrm{Ni}, \mathrm{Pb}$, and $\mathrm{Zn}$ in $\mathrm{HP}$ samples of various sampling sites have been given in Table-8. The Table-8 indicated significant positive correlation ofNi with $\mathrm{Zn}(\mathrm{r}=0.518), \mathrm{Cu}$ with $\mathrm{Fe}(\mathrm{r}=0.436), \mathrm{Zn}$ with $\mathrm{Fe}$ $(\mathrm{r}=0.392), \mathrm{Cu}$ with $\mathrm{Zn}(\mathrm{r}=.370)$, Fe with $\mathrm{Ni}$ $(\mathrm{r}=0.360)$ and $\mathrm{Pb}$ with $\mathrm{Zn}(\mathrm{r}=0.320)$. It was observed that almost all elements showed similar magnitude of contamination in various Union Councils of Taluka Sobhodero, District Khairpur, Sindh, Pakistan.

It has been further discussed that in study area, groundwater (HP and TW water) were being used for drinking, cooking and personal hygiene. Present study shows that in many area the concentration of As and $\mathrm{Fe}$ is higher than the recommended safe limits of WHO. This poses a serious problem for the local Government to protect human health from As threat. There are various form of arsenic pollution in water (Baig et al. 2007). Arsenic can combine with other elements to make chemicals used to preserve wood and to kill insects on cotton and other agricultural crops. High arsenic levels may come from certain fertilizers, animal feedlots, industrial waste and herbicides (Chakrabortiet al.2002). The As poisoning status in Sobhodero, Sindh, Pakistan, is at dangerous position; so millions of people are at arsenic risk Therefore, necessary preventive measures should be adopted to minimize the risk level in the study area.

\section{Conclusion}

The evaluation of total arsenic, copper, iron , nickel, lead and zinc contents in hand pump groundwater (243 samples) and tube-well groundwater (90 samples) of Sobhodero, Sindh, Pakistan, were performed in order to be aware about the arsenic and other elemental pollution in the study area. It was concluded that arsenic concentration in most of HP and TW samples was higher than the WHO permissible limits. The multivariate techniques, cluster analysis of understudy sites clearly showed the high, medium and less polluted sites for hand pump and tube-well groundwater samples. Generally, in the hand pump groundwater, the level of arsenic was higher than that of tube-well water possibly due to high depth. To reduce the impact of arsenic on human health there is now a need to have particular treatment systems to remove arsenic from drinking water.

\section{Recommendations}

More detailed understanding of local sources of arsenic and mechanisms of arsenic removal is required to be evaluated. More extensive studies would be required for building practical guidance on avoiding and reducing arsenic contamination especially in groundwater of Sobhodero, Sindh, Pakistan.

\section{Acknowledgement}

Authors are thankful to Higher Education Commission, Islamabad, and Government of Pakistan for financial support to complete this research work. Institute of Chemistry, Shah Abdul latif University Khairpur, is also acknowledged for providing peaceful environment to carry out present research work.

\section{References}

1. Abbas, M. \&Cheema, K. (2015). Arsenic levels in Drinking water and associated health risk in district Sheikhupura, Pakistan" The Journal of Animal \& Plant Sciences, 25, 719. 724.

2. Ali. J., Kazi, T.G., Baig, J.A.,Afridi,H.I.,et al (2015).Evaluation of the fate of arsenic-contaminated groundwater at different aquifers of Thar coalfield Pakistan. Environmental Science and Pollution Research, 22, 1925119263.

3. Arain, M.B., Kazi, T.G., Jamali, M.K., Jalbani, N. et al. (2007) "Total dissolved and bio-available elements in water and sediment samples and their accumulation in Oreochromismossambicus of polluted Manchar Lake, Chemosphere 70, 1845-1856.

4. Arain, M.B., Kazi, T.G., Baig, J.A.,Afridi, H.I.,et al. (2015) Co-exposure of arsenic and cadmium through drinking water and tobacco smoking: risk assessment on kidney dysfunction. Environmental Science and Pollution Research International, 22, 350-357.

5. Asadullah, M.N, \&Chaudhury, N. (2011).Poisoning the mind: Arsenic contamination of drinking water wells and 
children's educational achievement in rural Bangladesh. Economics of Education Review 30, 873-888.

6. Atta, R., Abida F.,Sajid M., \&Khadim H. (2016). "Arsenic in groundwater and its health risk assessment in drinking water of Mailsi, Punjab, Pakistan" Human and Ecological Risk Assessment: An International Journal 22, 187- 202.

7. Baig, J.A., Kazi, T.G., Arain, M.B., Afridi, H.I., et al. (2009). Evaluation of arsenic and other physico-chemical parameters of surface and ground water of Jamshoro, Pakistan. Journal of Hazardous , 166, 662-669.

8. Baig, J.A., Kazi, T.G. Shah, A.Q. Afridi, H.I., et al. (2011). Evaluation of toxic risk assessment of arsenic in male subject through drinking water in Southern Sindh Pakistan.Biological Trace Element Research. 143(2), 772-786

9. Baig J.A., Kazi T.G., Arain, M.B., Shah, A.Q., et al. (2010). Speciation and evaluation of Arsenic in surface and ground water: A multivariate case study. Ecotoxicology and Environmental Safety, 73(5) 914-923.

10. Chowdhury, S., Krause, A., Zimmermann, K.F. (2015)."Arsenic contamination of drinking water and mental health.IZA Discussion Paper No. 9400.

11. Chakraborti, D., Rahman, M.M., Chowdhury, U.K.,Paul, K., et al. (2002).Arsenic calamity in the Indian subcontinent, what lessons have been learned?Talanta58, 3-22.

12. Douillet, C., Currier, J., Saunders, J., Bodnar,W.M., et al. (2013)."Methylated trivalent arsenicals are potent inhibitors of glucose stimulated insulin secretion by murine pancreatic islets" Toxicology and Applied Pharmacology, vol. 267(1), 11-15.

13. Fakir, M.Y., Safayet, K.,Priyanka C., et al. (2016). "A Review of Groundwater Arsenic Contamination in Bangladesh" The Millennium Development Goal Era and Beyond" International Journal of Environmental Research and Public Health. 13(2), 215.

14. Find, R. (2001). National Primary Drinking Water Regulations; Arsenic and Clarifications to Compliance and New Source Contaminants Monitoring, Federal Register (FR), 22nd January, 66, 6976-7066.

15. Flanagan, S.V., Johnston, R,B.,\&Zheng, Y. (2012). Arsenic in tube well water in Bangladesh: health and economic impacts and implications for arsenic mitigation, Bulletin of the World Health Organization 90, 839-846.

16. Finkelman, R.B., Orem, W., Castranova, V., et al. (2002) Health impacts of coal and coal use: possible solutions. Int J Coal Geol. 50, 425-43.

17. Huang, Y. \&Chihiro I. (2016). Arsenic contamination of groundwater and agricultural soil irrigated with the groundwater in Mekong Delta, Vietnam" Environmental Earth Sciences(75) 757.

18. Hudak, P.F.(2000). Distribution and sources of arsenic in the southern high plains aquifer, Texas, USA. J Environ Sci Health A., 35, 899-913.

19. Hossain, M.D., Ahmed, H.U., Chowdhury, W.A., et al., (2014). Mental disorders in Bangladesh: a systematic review." BMC Psychiatry, 14:216.

20. Huang, L., Wu, L.H., \&Tsering, J.K. (2015) "The health effects of exposure to arsenic-contaminated drinking water: a review by global geographical distribution." International Journal of Environmental Health Research, 25, 432-452.

21. Ioannis, A.K., \&Athanasios A.K.(2006).Arsenic and other metal contamination of groundwater in the industrial area of Thessaloniki, Northern Greece.Environmental monitoring and assessment.123,393-406.

22. Jakhrani, M.A., Malik, K.M., Sahito, S., and A. A. Jakhrani, A.A. (2011)“ Analytical Investigation of Arsenic and Iron in Hand pumps and Tube-well Groundwater of Gambat, Sindh, Pakistan" Pak. J. Chem. 1 (3), 140-144.

23. Kamal, A.K., Bhabajitbhuyan andHariprasadsarma (2010). Lead, arsenic, fluoride, and iron contamination of drinking water in the tea garden belt of Darrang District, Assam, India. Environmental monitoring and assessment169,347-352.
24. Khan, S., Irfan, A., Shah, S.M., Riffat, N.M.\& Shah, M.T. (2015). Arsenic and Heavy Metal Concentrations in Drinking Water in Pakistan and Risk Assessment: A Case Study" Human and Ecological Risk Assessment, 21, 1020-1031.

25. Muhammad Qasim\&Mushtaque Ali Jakhrani (2017): Physicochemical and elemental contamination assessment in groundwater samples of Khairpur Mir's, Pakistan, Human and Ecological Risk Assessment: An International Journal, DOI: 10.1080/10807039.2016.1277415

26. M. Berg, St.P. Caroline, T.K.T. Pham, H.V. Mickey, L. Sampson, M. Leng, S. Samreth and D. Fredericks, Magnitude of arsenic pollution in the Mekong and Red River DeltasCambodia and Vietnam, Sci. Total Environ. 372 pp. 413-425, (2007).

27. Mandal BK and Suzuki KT. 2002. Arsenic round the world: A review. Talanta 58:201-35.

28. Mahar, M.T., Khuhawar, M.Y., Jahangir, T.M. \&Baloch, M.A. (2015). Determination of arsenic contents in groundwater of District Rahim Yar Khan Southern Punjab, Pakistan Arab J Geosci 8, 10983-10994.

29. Nickson, R.T., McArtur, J.M., Shrestha, B., et al. 2005 Arsenic and other drinking water quality issues, ApplGeochem Elsevier 20:55-68.

30. Phuong, N.M., Kang, Y., Sakurai, K.Sugihara, M.,Kien, C.N., et al. (2012).Antimony (Sb) and Arsenic (As) in Sb mining impacted paddy soil from Xikuangshan, China: differences in mechanisms controlling soil sequestration and uptake in Rice," Environmental Science and Technology, vol. 46, no. 6, pp. 3155-3162.

31. Phuong, N.M., Kang, Y., Sakurai, K., Sugihara, M., et al. (2012).Arsenic contamination in groundwater and its possible sources in Hanam, Vietnam.Environmental monitoring and assessment 184:4501-4515.

32. Rezende, P.S., Costa, L.M., Windmoller, C.C., (2013), Total and trivalent inorganic arsenic determination in water samples by selective hydride generation atomic absorption spectrometry. Br. J. Anal. Chem. 10:429-435.

33. Sadia,B., AbidaF.,Mehwish, R.\&Asif, J., (2015). Health risk of arsenic in the alluvial aquifers of Lahore and Raiwind, Punjab Province, Pakistan: an investigation for safer well water." Toxicological \& Environmental Chemistry, 97, 88807.

34. Seema, A.K., David, P.,Liaqat, A., \&Shah, M.T. (2016) "Arsenic exposure assessment from groundwater sources in Peshawar Basin of Khyber Pakhtunkhwa" Pakistan Journal of Himalayan Earth Sciences Volume 49, No. 1, pp.68-76.

35. Sidauruk, P., Cheng A.H.D., \&Ouazar, D. (1998) Ground water contaminant source and transport parameter identification by correlation coefficient optimization. Ground Water 36:208-214.

36. Sinha, J. B. \&Bishayee, A. (2013). "Nrf2-mediated redox signaling in arsenic carcinogenesis: a review," Archives of Toxicology, 87, 383-396.

37. Spayd, S.E., Robson, M.G., Xie, R., et al.(2012) Importance of Arsenic speciation in population exposed to arsenic in drinking water" HumEcol Risk Assess 18, 1271-91.

38. Stanger, G., Truong, V.T., Ngoc L.T.M.,Thanh, T.T. (2005).Arsenic in groundwater of the Lower Mekong.Environ Geochem Health, 27, 341-57.

39. Steven, E., Spayd, M.G., Robson, R. X.\& Brian T. B. (2012) "Importance of Arsenic Speciation in Populations Exposed to Arsenic in Drinking Water" Human and Ecological Risk Assessment, 18, 1271-1291.

40. Shrestha, B. (2002). Drinking water quality: future directions for UNICEF in Pakistan Consultancy Report 2 of 3, Water Quality, SWEET Project, UNICEF Pakistan, Islamabad.

41. Sullivan, R.J. (1969). National air pollution control administration Publication No. APTD 69-26, US Environmental Protection Agency, Raleigh, NC, pp. 60.

42. Thi T.G.L., Sthiannopkao, S.,and Kyoung-Woong K. (2009).Arsenic and other trace elements contamination in 
groundwater and a risk assessment study for the residents in the Kandal Province of Cambodia. Environment International (35), 455-460.

43. Toqeer Ahmed, Arshid Pervez, Muhammad Mehtab, Sikandar Khan Sherwani, (2015) Assessment of drinking water quality and its potential health impacts inacademic institutions of Abbottabad (Pakistan)" Desalination and Water Treatment 54, 1819-1828.

44. Vinod Kumar Gupta, et al. (2012). Arsenic speciation analysis and remediation techniques in drinking water" International Journal of Environmental Health Research Volume 40, - Issue 1-3.

45. Wang, Y.\&Shpeyzer, G.M.(1997). Genesis of thermal ground waters from Sippinan district, China.ApplGeochem $12,437-45$.

46. Wang, L., Kou, M.C., Weng, C.W., Hu, L.W., Wang, J.W., and $\mathrm{Wu}$, M.J. (2012) "Arsenic modulates heme oxygenase-1, interleukin-6, and vascular endothelial growth factor expression in endothelial cells: roles of ROS, NF $-\kappa B$, and MAPK pathways," Archives of Toxicology, 86(6), 879-896.

47. Welch, A.H., Westjohn, D.B., Helsel, D.R., et al. (2000). Arsenic in ground water of the United States-Occurrence and geochemistry. Ground Water 38, 589-604.

48. Yang Y.Y., Liu, L.Y., Guo, L.L.,Lv, Y.L., et al. (2015).Seasonal concentrations, contamination levels, and health risk assessment of arsenic and heavy metals in the suspended particulate matter from an urban household environment in a metropolitan city, Beijing, China Environmental monitoring and assessment187, 409.

49. Zivin J.G., \&Neidell, M. (2013)."Environment, health, and human capital. Journal of Economic Literature. 51(3), 689730 .

50. Zheng\&Ayotte, J.D. (2015). At the crossroads: hazard assessment and reduction of health risks from arsenic in private well waters of the northeastern United States and Atlantic Canada." Science of the Total Environment, 505, $1237-1247$. 